\title{
Perfil de lipoproteínas, triglicérides e glicose plasmáticos de pacientes com câncer durante o transplante de medula óssea
}

\author{
Plasma lipoproteins, triglycerides and glucose profile \\ of cancer patients during bone marrow transplantation
}

\author{
Adriana GARÓFOLO' \\ Patrícia Cláudia MODESTO ${ }^{1}$ \\ Letícia Navarro GORDAN, \\ Antonio Sérgio PETRILLI ${ }^{1}$ \\ Adriana SEBER ${ }^{1}$
}

RE S U M O

O objetivo deste estudo foi avaliar a evolução do perfil metabólico-nutricional de crianças e adolescentes com câncer que realizaram transplante de medula óssea. Dezoito pacientes submetidos a transplante de medula óssea foram avaliados prospectivamente de outubro de 2003 a agosto de 2004. A avaliação foi realizada por meio da análise bioquímica de sangue para albumina, lipídeos e glicose em três momentos: antes da infusão da medula óssea, após sete dias e após catorze dias do transplante de medula óssea. O teste de Friedman foi aplicado para comparar as distribuições nos períodos e o teste de Mann'Whitney para comparar as diferenças na evolução entre os grupos de transplante de medula óssea autólogo versus alogênico. Quinze dos dezoito pacientes foram elegíveis: sete portadores de leucemia, quatro de linfomas e quatro de tumores de células

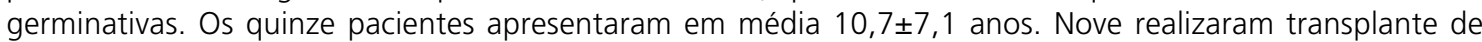
medula óssea autólogo e seis alogênico aparentado; dois utilizaram ciclosporina A como imunossupressor e três receberam irradiação corporal total como parte da terapia de condicionamento. Treze de quinze usaram nutrição parenteral. A média de internação foi 33 14 dias. As prevalências nos déficits de albumina e HDL-C aumentaram progressivamente durante o acompanhamento: $15 \%, 31 \%$ e $46 \%$ e $54 \%, 69 \%$ e $85 \%$, respectivamente. O mesmo ocorreu com os níveis de glicose e triglicérides, com aumento progressivo nas prevalências de anormalidades no decorrer do transplante de medula óssea, que foram de $7 \%, 43 \%$ e $50 \%$ e de $31 \%, 69 \%$ e $77 \%$, respectivamente. Níveis de colesterol total e de LDL-C acima do normal foram observados somente em um paciente no primeiro momento (antes da infusão da medula óssea). A análise da evolução das variáveis no decorrer dos três períodos demonstrou que os níveis de HDL-C, glicemia e triglicérides se modificaram significantemente. Quando se compararam pacientes que realizaram transplante de medula óssea alogênico versus autólogo, não foi possível detectar diferença estatisticamente significante. Porém os

\footnotetext{
1 Instituto de Oncologia Pediátrica, Serviço de Nutrição e Metabolismo, Departamento de Pediatria, Universidade Federal de São Paulo, Escola Paulista de Medicina. R. Botucatu, 743, 04023-062, São Paulo, SP, Brasil. Correspondência para/Correspondence to: A. GARÓFOLO.E-mail: <adrigarofolo@hotmail.com>.
} 
níveis de HDL-C e de triglicérides apresentaram alterações mais acentuadas nos pacientes que realizaram transplante de medula óssea alogênico. Os resultados sugerem que alterações metabólicas e bioquímicas ocorrem durante o período do transplante. Tais alterações, possivelmente, são multifatoriais, podendo estar associadas com o déficit nutricional, catabolismo protéico e distúrbios no metabolismo energético. Provavelmente, a desnutrição, o uso de nutrição parenteral, bem como a resposta inflamatória e as toxicidades dos medicamentos estejam implicadas como fatores causais desses distúrbios.

Termos de indexação: criança; adolescente; lipoproteínas; triglicérides; transplante de medula óssea.

\section{A B S T R A C T}

The objective was to evaluate the nutritional metabolic profiles in children and adolescents with cancer undergoing bone marrow transplants. Eighteen bone marrow transplantation patients were prospectively evaluated from October 2003 to August 2004. The assessment was based on the biochemical blood analyses of albumin, lipids and glucose at 3 different moments: before the bone marrow transplant, 7 days after the bone marrow transplant and 14 days after the bone marrow transplant. The Friedman test was performed to compare the distributions between the periods and the Mann' Whitney test to compare differences in the evolution between the bone marrow transplant groups: autologous versus allogeneic. Fifteen of the 18 patients were eligible: seven had leukemia, four lymphoma and four germ cell tumors. The mean age of the 15 patients was 10.7 \pm 7.1 years. Nine received autologous and six allogeneic-related bone marrow transplants; two used cyclosporin $A$ as an immunosuppressor and three total body irradiation as part of the conditioning regimen. Thirteen of the 15 received parenteral nutrition. The length of stay was $33 \pm 14$ days. The prevalence of albumin and HDL-C deficits increased progressively during follow up: $15 \%, 31 \%$ and $46 \%$ and $54 \%, 69 \%$ and $85 \%$, respectively. The same result was observed with the glucose and triglyceride levels, showing a progressive increase in the prevalence of abnormalities during bone marrow transplantation, with values of $7 \%, 43 \%$ and $50 \%$ and $31 \%, 69 \%$ and $77 \%$, respectively. Total cholesterol and LDL-C levels above the normal were only observed in one patient at the first moment (before the bone marrow transplant). The analysis of the evolution of the variables during the three periods demonstrated that the HDL-C, glucose and triglyceride levels changed significantly. No statistically significant differences were observed when comparing patients submitted to allogeneic and autologous bone marrow transplants. However, changes in the HDL-C and triglyceride levels were more accentuated in patients submitted to allogeneic bone marrow transplants. The results suggested that metabolic and biochemical changes occur during the period of bone marrow transplantation. These changes are probably multi-factorial, being associated with nutritional deficits, protein catabolism and disturbances in the energy metabolism. It is probable that malnutrition, the use of parenteral nutrition, as well as the inflammatory response to and toxicity of the drugs applied are implicated as causal factors of these abnormalities.

Indexing terms: child; adolescent; lipoproteins; triglycerides; bone marrow transplantation.

\section{N T R O D U Ç Ã O}

Transplante de medula óssea (TMO) ou transplante de células progenitoras hematopoiéticas é uma terapia reconhecida para uma variedade de doenças hematológicas, anormalidades genéticas e neoplasias. O procedimento é utilizado para restaurar a função da medula em pacientes que recebem quimioterapia e irradiação intensas, por meio da infusão de células progenitoras ou células-tronco (stem cells), com capacidade de multiplicação e diferenciação em todos os tipos de células sangüíneas maduras: eritrócitos, leucócitos e plaquetas ${ }^{1}$.

Quanto à origem das células, os transplantes podem ser autólogos - quando as células são originárias do próprio paciente - ou alogênicos - quando as células são doadas por um outro indivíduo. Caso o doador seja um gêmeo idêntico, o transplante é denominado singênico ${ }^{1,2}$.

As complicações do TMO podem ser agudas ou crônicas e dependem da doença de base e sua condição inicial antes do procedimento, 
do tipo de transplante, da quimioterapia preparatória e do regime de radioterapia. As principais complicações pós-transplantes incluem hemorragia, infecções, falência orgânica, doença do enxerto contra o hospedeiro $(\mathrm{DECH})$, falha ou rejeição do enxerto e doença recorrente ${ }^{2}$.

Além dessas complicações, o estado nutricional é fortemente afetado pelo processo do TMO. A oferta protéica reduzida, por exemplo, pode influenciar negativamente a função imunológica no período de estresse metabólico. Assim, estudos demonstraram a importância de ajustar as necessidades de energia para manter um balanço nitrogenado igual a zero ${ }^{3-5}$.

Pacientes que recebem TMO freqüentemente necessitam de nutrição parenteral, devido à redução da ingestão alimentar oral, associada às toxicidades do regime de condicionamento, principalmente em trato gastrintestinal5,6

Pacientes receptores de TMO alogênico recebem regimes de condicionamento com altas doses de quimioterapia, podendo ser combinada com irradiação corporal total para induzir imunossupressão profunda. A irradiação corporal é extremamente tóxica, induzindo à mucosite grave e prolongada ${ }^{7}$. Por isso, a nutrição parenteral total (NPT) tem sido a via mais utilizada para fornecer nutrientes durante a fase do TMO. Porém algumas circunstâncias podem limitar seu uso nesses pacientes. Entre elas, risco de infecção e distúrbios no metabolismo de lipídios e glicose. Dislipidemia e diabetes por uso de agentes imunossupressores, como a ciclosporina, são complicações freqüentes ${ }^{8}$, sendo que alguns autores acreditam que o seu uso é o fator mais importante associado à alta incidência de hipertrigliceridemia em pacientes receptores de TMO alogênico. Esse fator pode representar uma dificuldade no momento da decisão da oferta de lipídios e glicose endovenosos, pois níveis de triglicérides ou glicose significativamente elevados predispõem pacientes graves à falência ou disfunção orgânica, além do aumento do risco de infecção?.

O excesso de lipídio reduz a habilidade do sistema retículo-endotelial hepático de clarear bactérias durante o estado de estresse agudo, o que provoca aumento da fagocitose pelos macrófagos alveolares e aumento de infecção pulmonar em animais. Essa resposta se associa ao fato de que com o aumento da disponibilidade de triglicérides, os macrófagos tendem a fagocitá-los, em detrimento à fagocitose de bactérias ${ }^{10,11}$. Apesar disso, Lessen et al. ${ }^{12}$ não observaram aumento na incidência de infecções bacterianas ou fúngicas em pacientes TMO que receberam quantidades moderadas de lipídios endovenosos.

Aumento dos níveis de glicose circulante também pode prejudicar o sistema imune, pois inibe a atividade de monócitos. Em adultos, estudos demonstraram que níveis constantes acima de $220 \mathrm{mg} / \mathrm{dl}$ prejudicam elementos leucocitários fundamentais, reduzindo a aderência dos granulócitos, quimiotaxia, fagocitose e, conseqüentemente, a atividade microbicida ${ }^{9}$. Alterações desses mecanismos durante o estado crítico da doença têm sido associadas ao aumento de complicações orgânicas e infecciosas. Entretanto, a literatura é escassa quanto a informações referentes aos níveis plasmáticos de proteínas, lipídios e glicose em pacientes TMO. Por isso, o objetivo do estudo foi avaliar a evolução do perfil metabólico-nutricional (lipídios, glicídios e albumina) de pacientes com câncer durante o período do TMO.

\section{MÉ T O D O S}

Crianças, adolescentes e adultos jovens que necessitaram realizar TMO para tratamento de tumor maligno foram acompanhados durante o período de outubro de 2003 a agosto de 2004 no Instituto de Oncologia Pediátrica da Universidade Federal de São Paulo. Tratou-se de um estudo-piloto, cujas variáveis coletadas fazem parte da rotina do acompanhamento de pacientes submetidos a TMO. Todos os procedimentos e avaliações desses pacientes foram feitos com o seu consentimento e de seus pais ou responsáveis. Os participantes foram seguidos numa coorte prospectiva para avaliação do perfil bioquímico 
de sangue referente à albumina, lipídios e glicose. Essa avaliação realizou-se em três momentos: antes da infusão da medula óssea, após sete dias do TMO e após catorze dias do mesmo. Os seguintes valores de referência foram adotados: albumina inferior a 3,5g/d, lipoproteínas e lipídios (triglicérides, HDL-C, LDL-C) de acordo com o National Cholesterol Education Program ${ }^{13}$ e glicose superior a $110 \mathrm{mg} / \mathrm{dl}$. As amostras de sangue para análise laboratorial foram coletadas após jejum noturno de 12 horas, por meio de um cateter arterial, por meio de técnica anticoagulação durante a coleta. O colesterol sérico foi mensurado por método colorimétrico e a HDL-C e os triglicérides enzimaticamente (Sera-Pak ${ }^{\circledR}$ Plus). A LDL-C foi calculada de acordo com a equação de Friedwald ${ }^{14}$ : LDL-C= colesterol total - HDL-C - (triglicérides/5). Glucose Hexokinase II (GluHII) (Bayer) foi usada para determinar a glicose plasmática e o método turbimétrico para medir a albumina sérica. A história familiar foi coletada à admissão de cada paciente na unidade de TMO.

Como critérios de exclusão consideraram-se: coleta de dados inadequada ou ausência de informação, pacientes que ainda não haviam completado o período proposto para avaliação e pacientes que se recusassem a participar do estudo.

Foram realizadas análises de prevalência das alterações no perfil metabólico durante os períodos, evolução dos indicadores durante o período e comparação entre os grupos de TMO autólogo e alogênico. O teste de Friedman foi aplicado para comparar as distribuições nos períodos e o teste de Mann'Whitney para comparar as diferenças na evolução entre os grupos de TMO autólogo versus alogênico. O nível de significância foi estabelecido em $5 \%$ ou 0,05 .

\section{RESULTADOS}

Dezoito pacientes foram submetidos a $\mathrm{TMO}$, entretanto quinze foram elegíveis: sete portadores de leucemias, quatro de linfomas e quatro de tumores de células germinativas. A faixa etária dos quinze pacientes apresentou mediana de 9,8 anos $(1,8-25,3)$ e média de $10,7 \pm 7,1$ anos; em relação ao sexo, havia oito crianças (seis do sexo feminino e dois do masculino) e sete adolescentes e adultos jovens (quatro do sexo feminino e três do masculino).

Nove realizaram TMO autólogo (60\%) e seis, alogênico (40\%) aparentado; dois utilizaram ciclosporina $\mathrm{A}$ e o restante metotrexato como imunossupressor e três pacientes que realizaram TMO alogênico receberam irradiação corporal total como parte da terapia de condicionamento. Treze dos quinze pacientes analisados usaram NPT durante o período do TMO.

O período de internação dos pacientes para a realização do TMO até sua estabilização clínica e alta ou morte foi de $33 \pm 14$ dias em média, com mediana de 30 dias (13-71). Três dos quinze pacientes analisados foram a óbito após o acompanhamento, durante a internação. Por motivo de alta hospitalar no sétimo dia após o $\mathrm{TMO}$, bem como problemas com a coleta do exame, alguns pacientes não foram analisados para todas as variáveis bioquímicas propostas: glicose $(n=14)$, triglicérides ( $n=13), \operatorname{HDL}-C(n=13)$ e albumina $(n=13)$. As prevalências nos déficits de albumina e HDL-C aumentaram progressivamente durante o acompanhamento: 15\%, 31\% e $46 \%$ e $54 \%$, $69 \%$ e $85 \%$, respectivamente. O mesmo ocorreu com os níveis de glicose e triglicérides, com aumento progressivo nas prevalências de anormalidades no decorrer do TMO, que foram de $7 \%$, $43 \%$ e $50 \%$ e de $31 \%$, $69 \%$ e $77 \%$, respectivamente. Níveis de colesterol total e de LDL-C acima do normal foram observados somente em um paciente no primeiro momento (antes da infusão da medula óssea). A análise da evolução das variáveis no decorrer dos três períodos demonstrou modificações significantes nos níveis de HDL-C, glicemia e triglicérides (Figura 1).

Quando se compararam pacientes que realizaram TMO alogênico com os que realizaram o autólogo, não foi possível detectar diferenças estatisticamente significantes. Porém, os níveis de HDL-C e de triglicérides apresentaram alterações mais acentuadas nos pacientes que realizaram TMO alogênico (Figura 2). 

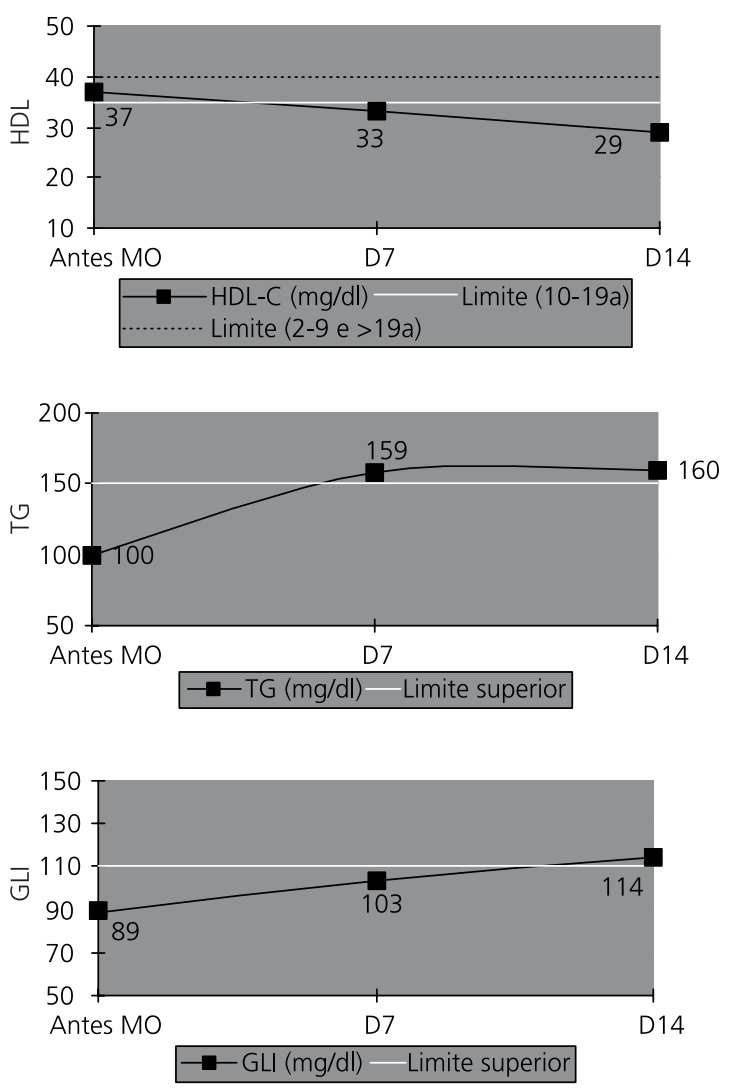

Figura 1. Evolução dos níveis de HDL-C, triglicérides (TG) e glicose (GLI) (mediana) durante os três períodos do estudo. HDL-C: $p<0,04 ;$ TG: $p<0,04$; GLI: $p<0,05$ (teste de Friedman) Médias: HDL-C: $40 \pm 11,33 \pm 11,27 \pm 12$; TG: $111 \pm 68,158 \pm 65$, $172 \pm 72$; GLI: $92 \pm 24,122 \pm 46,118 \pm 37$
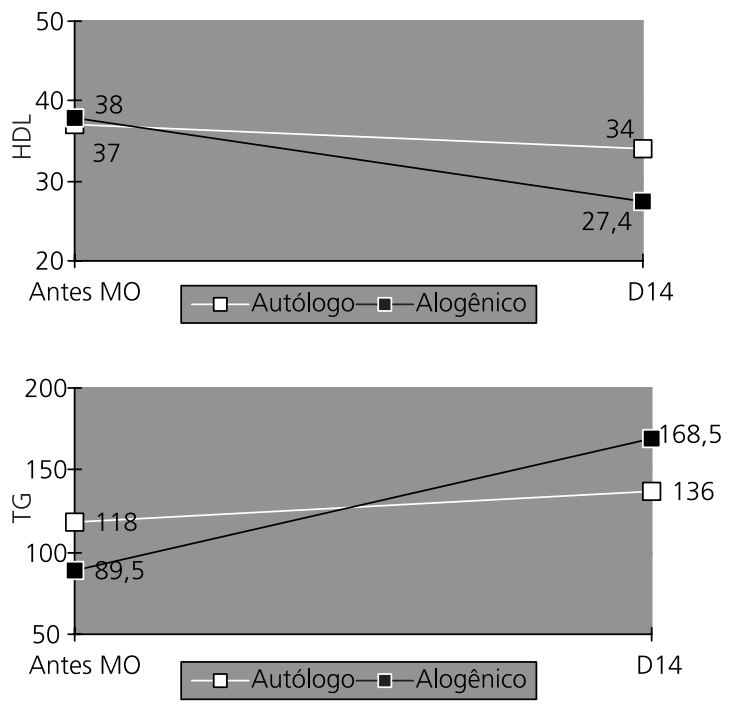

Figura 2. Comparação das medianas do HDL-C e triglicérides (TG) durante o período de acompanhamento (antes do TMO e no dia 14).

Teste de Mann'Whitney: TMO autólogo versus alogênico; $p>0,05$ (não significante).
D I S C U S S Ã O

Os resultados desta pesquisa sugerem que alterações metabólicas e bioquímicas ocorrem durante o período do TMO. A maioria dos pacientes neste estudo (73\%) era portadora de doenças hematológicas malignas, enquanto que o restante, $27 \%$, tinha tumores de células germinativas.

Halton et al. ${ }^{15}$ demonstraram alterações do perfil lipídico em crianças com leucemia linfóide aguda ao diagnóstico e após a terapia de remissão com L-asparaginase. Estudos anteriores observaram anormalidades lipídicas significativas em pacientes com leucemias e linfomas, particularmente níveis baixos de HDL-C e elevados de triglicérides $^{16,17}$. Entretanto, níveis baixos de HDL-C também foram encontrados em crianças com outras doenças malignas disseminadas ${ }^{15}$. Como pacientes candidatos a TMO, geralmente, apresentam-se em remissão da doença maligna, a presença de tumor pode não estar relacionada com as alterações observadas.

Este estudo não avaliou um grupo-controle de indivíduos saudáveis para comparar os dados. Entretanto, comparando-se os níveis de HDL-C observados nos pacientes receptores de TMO com aqueles de três estudos com crianças e adolescentes normais da população brasileira $52 \mathrm{mg} / \mathrm{dl}^{18}, 49 \mathrm{mg} / \mathrm{dl}^{19}$ e $50 \mathrm{mg} / \mathrm{dl}$ para meninos e $46 \mathrm{mg} / \mathrm{dl}$ para meninas ${ }^{20}$, respectivamente -, podemos concluir que os pacientes TMO apresentaram médias inferiores, principalmente nos dois últimos períodos. Da mesma forma, os níveis de triglicérides apresentaram-se mais elevados nos indivíduos submetidos a TMO deste estudo, quando comparados às crianças e adolescentes do estudo de Lima et al. ${ }^{20}$, que observaram $55 \pm 16 \mathrm{mg} / \mathrm{dl}$ para o sexo masculino e $82 \pm 25 \mathrm{mg} / \mathrm{dl}$ para o feminino.

Alguns autores sugerem que alterações metabólicas ocorrem durante o TMO, com aumento do gasto energético. Essas alterações podem ser decorrentes do estresse metabólico provocado pela terapia e procedimentos do TMO, similarmente à resposta inflamatória ocasionada 
em outras circunstâncias como cirurgias, trauma, câncer e sépsis ${ }^{21}$. Durante a resposta inflamatória há aumento na produção de citocinas pró-inflamatórias responsáveis pelas alterações metabólicas, entre elas, hiperglicemia, hipertrigliceridemia e redução na síntese de albumina ${ }^{22}$.

Comparando pacientes receptores de TMO autólogo versus alogênico quanto ao gasto energético de repouso (GER), estimado por calorimetria, Chamouard et al. ${ }^{23}$ demonstraram que o GER foi significantemente diferente durante a fase de aplasia. Houve um aumento de $11 \%$ no GER entre o grupo de receptores de TMO autólogo e redução do GER de 7\% a 9\% entre o grupo de TMO alogênico em relação ao gasto energético calculado por equações ${ }^{23}$. Isso poderia sugerir diferenças entre os tipos de TMO com relação à resposta metabólica frente ao TMO.

Neste estudo, apesar de a análise estatística entre TMO autólogo e alogênico não ter demonstrado diferenças significantes, os níveis de HDL-C e de triglicérides apresentaram maiores alterações nos pacientes que realizaram TMO alogênico.

Receptores de TMO alogênico apresentam maior escore de mortalidade, sugerindo que esse grupo seja de maior risco ${ }^{24}$. Esses pacientes recebem regimes de condicionamento com altas doses de quimioterapia, combinada com irradiação corporal total para induzir imunossupressão profunda. Essa terapia provoca um tempo prolongado de neutropenia e aumenta o intervalo da recuperação hematopoiética da medula, o que torna o paciente mais suscetível a infecções? ${ }^{7}$. Provavelmente, esse tipo de TMO esteja mais associado a uma resposta inflamatória mais intensa e, portanto, a maiores alterações no metabolismo energético, particularmente de lipídios.

Alguns autores têm sugerido que o uso de ciclosporina A, em pacientes receptores de TMO alogênico, pode ser o fator mais associado à alta incidência de hipertrigliceridemia ${ }^{9}$. Entretanto, apenas dois pacientes acompanhados no estudo usaram ciclosporina $A$, o que descarta essa possibilidade como causa das alterações nos demais indivíduos.
Neste estudo, acreditamos que tais alterações, possivelmente, sejam multifatoriais, podendo estar associadas ao déficit nutricional, catabolismo protéico e distúrbios no metabolismo energético. Provavelmente, a desnutrição, o uso de nutrição parenteral, bem como a resposta inflamatória e as toxicidades dos medicamentos estejam implicados como fatores causais desses distúrbios.

Apesar de os valores medianos de albumina não terem demonstrado alterações significantes no decorrer do período $(3,9,3,6$ e $3,5 \mathrm{mg} / \mathrm{dl} ; p>0,10)$, as taxas de pacientes com déficit aumentaram progressivamente. Tais mudanças, que ocorreram concomitantemente ao aumento dos níveis de triglicérides e glicose e redução nos níveis de HDL-C, podem estar mais associadas à resposta pró-inflamatória do que às alterações do estado nutricional. A albumina tem sido apontada como uma proteína negativa de fase aguda da resposta inflamatória, demonstrando valor prognóstico ${ }^{25}$.

Independentemente da causa, pacientes com níveis de glicemia e triglicérides significativamente elevados são mais suscetíveis a apresentarem falência ou disfunção orgânica associada. O excesso de lipídio circulante pode prejudicar o clareamento de bactérias pelas células de Kupffer durante o estado de estresse agudo, predispondo ao aumento de infecções pulmonares devido ao aumento da fagocitose nos macrófagos alveolares ${ }^{10,11,26}$. O aumento da glicose circulante também causa prejuízos na imunidade, pois inibe a atividade de monócitos e prejudica a atividade leucocitária, reduzindo a aderência dos granulócitos, quimiotaxia, fagocitose e, conseqüentemente, a atividade microbicida9 .

Por outro lado, a molécula de HDL-C é um transportador periférico de colesterol para o fígado; além dessa função, tem importantes implicações na proteção contra os danos peroxidativos. A molécula de HDL-C protege a LDL-C contra a peroxidação via paraoxonase e a redução da glutationa peroxidase dependente de selênio. A partícula de HDL-C também é capaz de varrer 
diretamente radicais livres de oxigênio, além de outras funções ${ }^{27}$. Como vários mecanismos estão associados ao aumento do estresse oxidativo em pacientes com câncer, alterações na HDL-C, provavelmente, desempenhem papel importante na patogênese das toxicidades orgânicas ${ }^{28-30}$.

Como alterações desses mecanismos durante o estado crítico da doença podem estar associadas ao maior número de complicações orgânicas e infecciosas, estudar essas moléculas e as apoproteínas, elucidando os fatores associados a tais alterações, bem como suas implicações no prognóstico de pacientes TMO, deveria ser o objetivo de futuros estudos.

\section{REFER Ê NCIAS}

1. Serber A. Transplante de células progenitoras em pediatria. Pediat Mod. 1999; 35(8):630-4.

2. Lozano JE, Cuéllar F. Transplante de médula ósea. Revisión de actualidad. Acta Med Colomb. 1991; 16(6):322-32.

3. Szeluga DJ, Stuart RK, Brookmeyer R, Utermohlen $\mathrm{V}$, Santos GW. Energy requirements of parentelly fed bone marrow transplant recipients. JPEN. 1985; (9):139-43.

4. Geibig B, Owens JP, Mirtallo JM. Parenteral nutrition for marrow transplant recipients: evaluation of an increased nitrogen dose. JPEN. 1991; 15(2):184-8.

5. Weisdorf SS, Schwarzenberg. Nutritional support of hematopoietic stem cell recipients. In: Thomas DE, Blume KG, Forman SJ, editores. Hematopoietic cell transplantation. Malden (USA): Blackwell Science; 1999.

6. Stiff P. Mucositis associated with stem cell transplantation: current status and innovative appoaches to management. Bone Marrow Transplant. 2001; 27(Suppl 2):S3-S11.

7. Muscaritoli M, Grieco G, Capria S, lori AP, Fanelli FR. Nutritional and metabolic support in patients undergoing bone marrow transplantation. Am J Clin Nutr. 2002; 75(2):183-90.

8. Papadoupoulou A. Nutritional considerations in children undergoing bone marrow transplantation. Eur J Clin Nutr. 1998; 52(12): 863-71.

9. Hasse J, Roberts S. Transplantation. In: Rombeau $\mathrm{JL}$, Rolandelli RH, editores. Clinical nutrition parenteral nutrition. Philadelphia: WB Saunders; 2001.

10. Aksnes J, Eide TJ, Nordstrand K. Lipid entrapment and cellular changes inthe rat, lung and liver after long-term parenteral nutrition with lipid emulsion. APMIS. 1996; 104(7-8):515-22.

11. Perez-Jaffe LA, Fufth EE, Minda JM, Unger LD, Lawton TJ. Massive macrophage lipid accumulation presenting as hepatoesplenomegaly and lymphadenopaty associated with long-term total parenteral nutrition therapy for short bowel syndrome. Hum Pathol. 1998; 29(6):651-5.

12. Lessen $P$, Bruemmer BA, Bowden RA, Gooley $T$, Aker SN, Mattson D. Intravenous lipid dose and incidence of bacteremia and fungemia in patients undergoing bone marrow transplantation. Am J Clin Nutr. 1998; 67(5):927-33.

13. National Colesterol Education Program. The expert panel on blood colesterol levels in children and adolescents. Pediatrics. 1992; 89(Suppl 3):525-70.

14. Friedewald WT, Levy RI, Fredrickson DS. Estimation of the concentration of low-density lipoprotein cholesterol in plasma, without use of the preparative ultracentrifuge. Clin Chem. 1972; 18(6):499-502.

15. Halton JM, Nazir DJ, Matthew JM, Barr RD. Blood lipid profiles in children with acute lymphoblastic leukemia. Cancer. 1998; 83(2):379-84.

16. Spiegel RJ, Schaefer EJ, Magrath IT, Edwards BK. Plasma lipid alterations in leukemia and lymphoma. Am J Med. 1982; 72(5):775-82.

17. Blackman JD, Cabana VG, Mazzone T. The acutephase response and associated lipoprotein abnormalities accompanying lymphoma. J Int Med. 1993; 233(2):201-4.

18. Gerber ZRS, Zielinsky P. Fatores de risco de aterosclerose na infância: um estudo epidemiológico. Arq Bras Cardiol. 1997; 69(4):231-6.

19. Moura EC, Castro CM, Mellin AS, Figueiredo DB. Perfil lipídico em escolares de Campinas, SP, Brasil. Rev Saúde Pública. 2000; 34(5):499-505.

20. Lima SCVC, Arrais RF, Almeida MG, Souza ZM, Pedrosa LFC. Plasma lipid profile and lipid peroxidation in overweight or obese children and adolescents. J Pediatr. 2004; 80(1):23-8.

21. Ringwald-Smith K, Williams R, Horwitz E, Schmidt $M$. Determination of energy expenditure in bone marrow transplant patient. NPC. 1998; 13: 215-8.

22. Raguso CA, Dupertuis YM, Pichard C. The role of visceral proteins in the nutritional assessment of intensive care unit patients. Curr Opin Clin Nutr Metab Care. 2003; 6(2):211-6. 
23. Chamouard CV, Chambrier C, Michallet $M$, et al. Energy expenditure during allogeneic and autologous bone marrow transplantation. Clin Nutr. 1998; 17(6):253-7.

24. Schneider DT, Lemburg P, Sprock I, Heying R, Gobel $U$, Nurnberger W. Introduction of the oncological pediatric risk of mortality score (O-PRISM) for ICU support following steam cell transplantation in children. Bone Marrow Transplant. 2000; 25(10): 1079-89.

25. Marín LA, Salido JA, López A, Silva A. Preoperative nutritional evaluation as a prognostic tool for wound healing. Acta Orthop Scand. 2002; 73(1):2-5.

26. Hamawy K, Moldawer LL, Georgieff M, Valicenti AJ, Babayan VK, Bristian BR, et al. The effect of lipid emulsions on reticuloendothelial system function in injured animal. JPEN. 1985; 9(5):559-65.
27. Stein O, Stein Y. Atheroprotective mechanisms of HDL. Atherosclerosis. 1999; 144(2):285-301.

28. Follézou JY, M Bizon. Cancer chemotherapy induces a transient increase of serum-iron level. Neoplasma. 1986; 33(2):225-31.

29. Schmidt $K$. Interation of Antioxidative Micronutrients with Host Defense Mechanisms. A Critical Review. Int J Vit Nutr Res. 1997; 67(5): 307-11.

30. Oldham KM, Bowen PE. Oxidative stress in critical care: is antioxidant supplementation beneficial? J Am Diet Assoc. 1998; 98(9):1001-8.

Recebido em: 30/3/2005

Versão final reapresentada em: 24/6/2005 Aprovado em: 2/8/2005 